\title{
Dispersal timing and drought history influence the response of bacterioplankton to drying- rewetting stress
}

\author{
Anna J Székely and Silke Langenheder \\ Department of Ecology and Genetics/Limnology, Uppsala University, Uppsala, Sweden
}

\begin{abstract}
The extent and frequency of drought episodes is expected to increase in the following decades making it a crucial stress factor for smaller water bodies. However, very little is known about how bacterioplankton is affected by increased evaporation and how these communities reassemble after rewetting. Here, we present results from a microcosm experiment that assessed the effect of dryingrewetting stress on bacterioplankton in the light of the stress history and the rate and timing of dispersal after the rewetting. We found that the drying phase resulted mainly in a change of function, whereas the complete desiccation and rewetting processes strongly affected both composition and function, which were, however, influenced by the initial conditions and stress history of the communities. Effects of dispersal were generally stronger when it occurred at an early stage after the rewetting. At this stage, selective establishment of dispersed bacteria coupled with enhanced compositional and functional recovery was found, whereas effects of dispersal were neutral, that is, predictable by dispersal rates, at later stages. Our studies therefore show that both the stress history and the timing of dispersal are important factors that influence the response of bacterial communities to environmental change and stress events.
\end{abstract}

The ISME Journal (2017) 11, 1764-1776; doi:10.1038/ismej.2017.55; published online 25 April 2017

\section{Introduction}

Current climate trends indicate increasing frequency of drought episodes in many parts of the world (Humphries and Baldwin, 2003; Jiménez Cisneros et al., 2014). Accordingly, temporary freshwater ecosystems that are characterized by recurrent drying-rewetting cycles have been receiving increasing attention. The effect of drought on microbial communities in aquatic systems has mostly been studied in sediments and biofilms (Amalfitano et al., 2008; Marxsen et al., 2010; Timoner et al., 2012; Pohlon et al., 2013; Proia et al., 2013; Timoner et al., 2014), and less focus has been given to the effects on bacterioplankton (Fazi et al., 2008, 2013). Drought episodes also represent an ideal setting to explore the effect of stress episodes from a community ecology perspective (Lake, 2003; Chase, 2007; Barthès et al., 2015).

Drying-rewetting cycles are relatively harsh ecological perturbations that comprise three main consecutive phases (Figure 1): (1) the drying process, (2) the desiccated phase when the environment is

Correspondence: AJ Székely, Department of Ecology and Genetics/ Limnology, Uppsala University, Norbyvägen 18D, Uppsala 75236, Sweden.

E-mail: anna.szekely@ebc.uu.se

Received 11 November 2016; revised 21 February 2017; accepted 3 March 2017; published online 25 April 2017 completely dry and (3) the rewetting or rehydration process. For microbial communities, all these steps represent selective forces. During the drying process water gradually evaporates resulting in the increase of electrical conductivity (Ec) and nutrient concentration, and in compositional changes of dissolved organic matter (Boven et al., 2008; Vazquez et al., 2010; Ylla et al., 2010; Zoppini et al., 2010). Bacterial communities may either be unaffected by this process (i.e., resistant) or show certain level of sensitivity, resulting in either only compositional changes (i.e., functional redundancy) or only functional changes (i.e., functional plasticity) or both (Allison and Martiny, 2008; Comte and del Giorgio, 2011). Meanwhile, the individual members of the bacterial community can respond in three main ways to such stress events: they may adapt through phenotypic plasticity and keep the abundance of their active cells essentially unchanged (resistant), they can fail to adapt and be negatively affected (sensitive), or they may 'take advantage' of the new conditions and increase in activity and abundance (opportunist) (Evans and Hofmann, 2012; Shade et al., 2012).

At the end of the drying phase, bacteria have to form inactive dormant cells to survive the subsequent desiccation period (Schimel et al., 2007; Lennon and Jones, 2011). The ability of dormancy is considered to be a bet-hedging strategy that is achieved by stochastic switching between different 


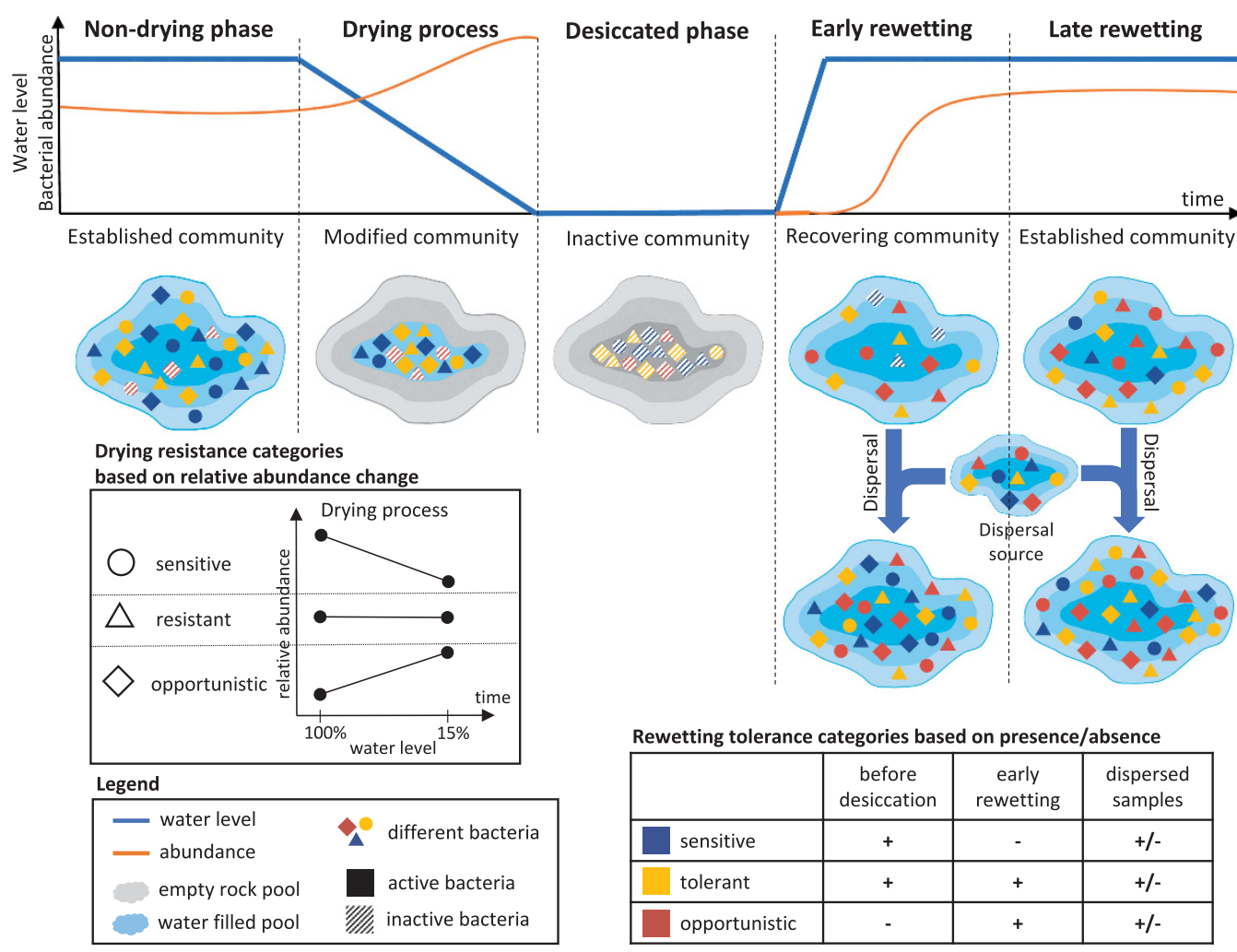

Figure 1 The drying-rewetting cycle and the response of the different stress tolerance groups. The upper part of the figure represents the changes in water level (blue line) during an ideal drying-rewetting cycle as well as the expected changes in bacterial abundance (orange line). The middle part of the figure shows the effect of the process on the rock pools and their bacterial communities. The geometric symbols (circles, triangles and rhomboids) represent bacterial OTUs with different stress response strategies. The shape of the symbols relates to different response of the OTUs to the drying stress (i.e., increased evaporation of water). The drying stress response categories were determined based on the changes of relative abundance of each OTU. The colour of the symbols represents the rewetting tolerance groups, which were defined based on the presence-absence of the OTUs through the steps of the rewetting process. Active bacteria are fully coloured, while inactive bacteria are striped. In this study only active bacteria were measured. The right side of the figure shows the effect of dispersal from an undisturbed source at two different time points after the rewetting (i.e., early after rewetting when the recipient community is less established and later when the recipient community is more established).

physiological states (Beaumont et al., 2009). During the rewetting process, nutrients and dissolved organic matter are resuspended in the water body (Ylla et al., 2010) together with the dormant cells that awake to colonize the reformed aquatic environment. The awakening from dormancy is considered to be a stochastic process (Buerger et al., 2012), which in the initial stages of recovery following an inactive period can lead to the assembly of divergent communities (Lee et al., 2017) that are strongly affected by priority effects (Fukami, 2015). However, as long as the conditions stabilize the initial communities are expected to converge through a succession of microbial species (Fazi et al., 2008) and eventually recover to stable communities similar to the ones before the stress event (Lee et al., 2017).

It has been demonstrated that consecutive dryingrewetting cycles select for macroinvertebrates with traits that help drought resistance (Brock et al., 2003; Chase, 2007; Bogan and Lytle, 2011). For bacteria there are several examples that show that disturbance history and past environmental conditions leave their imprint on the composition and function of bacterial communities (Bressan et al., 2008;
Hawkes and Keitt, 2015). Furthermore, in the case of soil bacteria, it has been shown that intensified exposure to rainfall (i.e., larger rainfall events separated by longer dry periods) increases the relative abundance of drying-rewetting-tolerant traits (Evans and Wallenstein, 2014). Consequently, the drying-rewetting history of a given aquatic habitat is expected to influence the resistance and recovering capacity of its bacterial communities against subsequent drying-rewetting episodes.

Dispersal can also influence the stress response of bacterial communities and enhance its compositional and functional resilience (Shade et al., 2012; Griffiths and Philippot, 2013). In the case of complex stress episodes such as drying-rewetting, dispersal can enhance recovery by acting as an additional source of taxa beside tolerant taxa that survive desiccation in seed banks. For microorganisms, dispersal is often considered as a stochastic process, which means that the abundance of a taxon in a local community can be predicted from its abundance in the regional species pool and the rate of dispersal (Hubbell, 2001; Sloan et al., 2006; Östman et al., 2010). However, dispersed propagules also have to 
1766

withstand the abiotic and biotic pressures and efficiently exploit the resources of the recipient habitat to successfully establish and propagate at the new locality (Hanson et al., 2012). Hence, the outcome of dispersal processes depends also on the complexity of the local communities (Mallon et al., 2015), and priority effects, where dispersal timing and arrival order of species define the final composition of the recipient community (Fukami, 2015). Consequently, Vellend et al. (2014) recently defined dispersal as a process that can influence communities in both stochastic and deterministic ways depending on the dispersal rate and the state of the recipient community. Particularly, they propose that the predictability of the outcome of a dispersal event depends on whether the recipient habitat is 'empty' or populated by an established community on which local selection already had time to operate.

Following a harsh stress event such as a dryingrewetting episode, communities have to be essentially reassembled from the local inactive seed bank (Figure 1). During the initial phase of recovery, the awaken, previously dormant local species undergo a secondary succession process driven by local selective forces until a complex, stable community is formed (Fierer et al., 2010). Hence, at early stages of the community recovery we anticipate that the establishment success of the propagules that arrive via dispersal will be strongly affected by the ongoing succession processes, and therefore the outcome of such dispersal event will be poorly predictable by solely its magnitude (i.e., dispersal rate). On the contrary, at later stages of community formation, when the recipient community is more stable, we expect the establishment of dispersed bacteria to be principally stochastic, which makes its outcome more predictable based on the rate of dispersal.

Here we present a microcosm experiment where we used three temporary water bodies (rock pools) differing in the frequency of historical drought events. Water from all source pools were subjected to a drying-rewetting cycle under controlled conditions to examine its effects on heterotrophic bacterial community composition and function. We also tested how dispersal aids recovery after rewetting and how dispersed cells establish in the target community depending on the timing of dispersal. We hypothesized that (1) the extent of resistance and resilience of the bacterial community to the drying-rewetting cycle will depend on past events, that is, frequency of previous drying-rewetting cycles; (2) dispersal will facilitate the recovery of the bacterial communities to the state prior the stress episode; (3) dispersal timing will influence the effect of dispersal on stress recovery.

\section{Materials and methods}

Experimental design

Water samples. On 12 September 2010, water samples were collected from rock pools at
Uggelhällorna peninsula ( $\mathrm{N} \mathrm{60} 39.895^{\prime}$, E 18²5.836') located on the Gräsö island along the Swedish Baltic Sea Coast (Langenheder et al., 2012; Székely and Langenheder, 2014). Three freshwater pools were chosen that differed in their size and therefore the probability/frequency of drying-rewetting episodes. The biggest pool was an $\sim 1 \mathrm{~m}$ deep permanent pool (P), which never dried up during our previous studies in the area (Langenheder et al., 2012; Székely and Langenheder, 2014). The smallest pool was a temporary pool $(\mathrm{T})$, which contained water only for few days after raining, while the third pool was a medium sized pool (M), which dried up only during longer rainless periods. The three pools were located within an area of $100 \mathrm{~m}^{2}$ but were not directly connected to each other.

Twenty litres of water from each pool were collected and transferred immediately to the laboratory, where they were filtered through 1.2- $\mathrm{mm} 142 \mathrm{~mm}$ Type A/E glass filters (Pall Corporation, Port Washington, NY, USA) to remove phytoplankton to be able to incubate the microcosms in dark and focus on heterotrophic bacterial communities. The water was stored at room temperature and within 2 days after sampling we set up a microcosm experiment in three replicates to test both the effect of a drying-rewetting episode and the effect of dispersal on the rewetted communities.

\section{Drying phase}

To test the effect of drying, the first phase of each microcosm experiment comprised of three open plastic boxes $\left(27 \times 39 \times 19 \mathrm{~cm}^{3}\right)$-one per replicate for each sampled rock pool (P, M, T)-filled with $2.5 \mathrm{~L}$ mix of water and $10 \%\left(\mathrm{~V} \mathrm{~V}^{-1}\right)$ 4-mm biofilm carrier expanded clay beads (Biolite, Degrémont, Paris, France; Figure 2). The microcosms were incubated in a dark, constant temperature room at $22{ }^{\circ} \mathrm{C}$ and $28 \%$ relative humidity, where the water evaporated at a slow continuous rate $\left(\sim 4 \%\left(\mathrm{~V} \mathrm{~V}^{-1}\right)\right.$ per day). Samples for bacterial abundance, bacterial respiration, dissolved organic carbon (DOC) and nutrient content were taken at the beginning of the incubation (initial sampling: day 0, 100\% water level) and when $85 \%$ of water evaporated from the microcosms (drying sampling: day 20, 15\% water level). At this point, samples were taken to estimate the functional diversity and bacterial community composition during the drying phase. Conductivity was monitored at several additional time points through the drying process. On day 24, 4 days after the drying sampling all boxes were completely dry and the desiccated beads were stored in sterile containers at room temperature until rewetting. To distinguish the effect of the drying stress from other effects related to the incubation conditions, a set of control microcosms that also contained $10 \%\left(\mathrm{~V} \mathrm{~V}^{-1}\right)$ of beads but were covered with a lid to avoid evaporation was incubated in parallel with the 


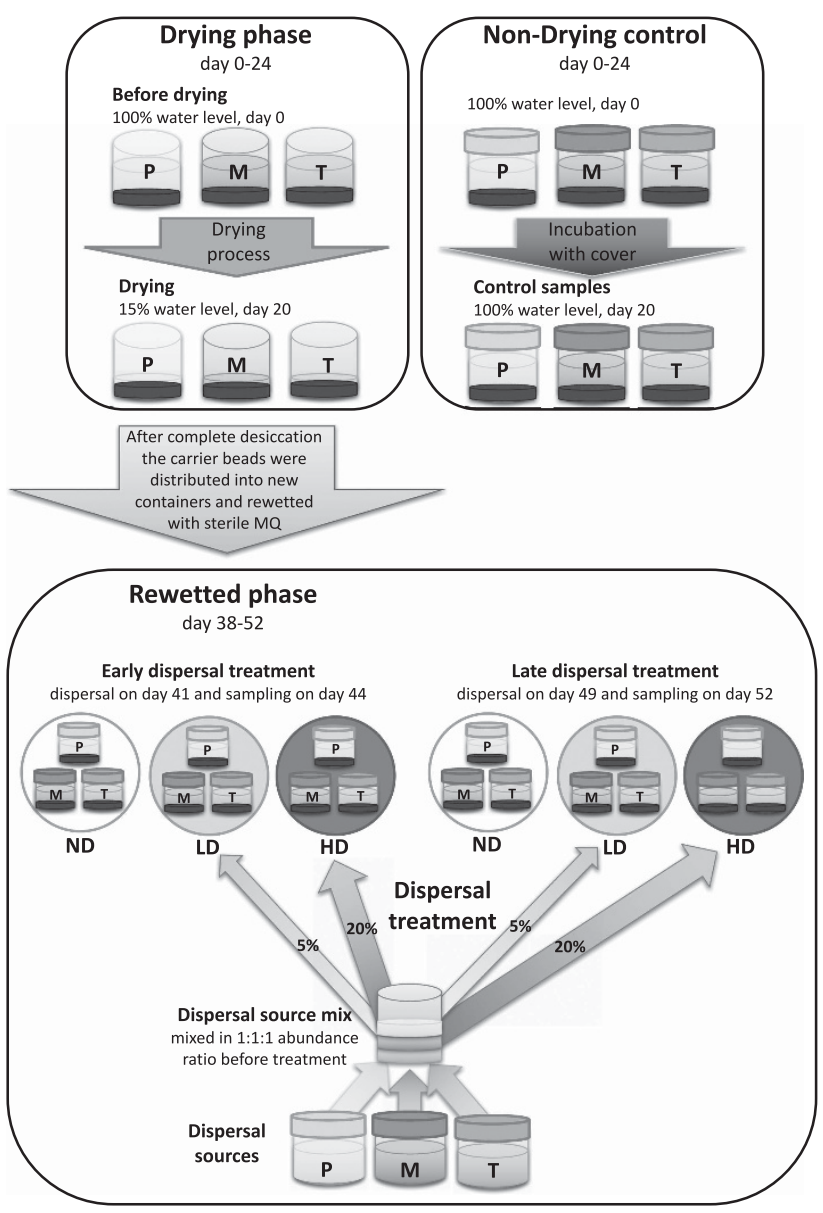

Figure 2 Experimental setup. $\mathrm{P}$ represents incubations using water from the permanent rock pool, $\mathrm{M}$ represents the medium and $\mathrm{T}$ the temporary rock pool. ND indicates no dispersal treatment, LD indicates low (5\%) and HD high (20\%) dispersal rate treatment, respectively. The set-up presented here was carried out with triplicates.

drying samples and were also sampled on day 20 (control sampling; Figure 2).

\section{Rewetting}

On day 38, two weeks after the complete evaporation of water, the desiccated beads from each microcosm were distributed into six new microcosms, rewetted with autoclaved MQ water to reach similar conductivity as the ones measured in the initial samples before drying. These rewetted beads were incubated under the same conditions as during the drying phase, but this time with closed lids to avoid evaporation (Figure 2: Rewetted phase). Half of the new microcosms were used to test the effect of a dispersal event that occurred early after the rewetting (3 days after rewetting, on day 41; called 'early dispersal treatment' throughout the manuscript). The other half of the new microcosms was used to test the effect of a dispersal event that occurred at a later stage after the rewetting (11 days after rewetting, on day 49; called 'late dispersal treatment' throughout the manuscript).
Dispersal

For both the early and late dispersal treatment three different levels of dispersal rates were applied: high dispersal (HD), where $20 \%$ of the cells were exchanged with cells from the dispersal source; low dispersal (LD), where $5 \%$ of the cells were exchanged; and no dispersal (ND) (Figure 2). For each replicated microcosm experiment, the dispersal source consisted of water samples from each original pool that were stored under the same temperature condition than the experimental treatments and mixed in equal abundance ratio before each dispersal event (dispersal source mix; Figure 2). The cell exchange was conducted by replacing $20 \%$ or $5 \%$ of the cells in the high or low dispersal microcosms, respectively. For this, cells from the dispersal source mix were collected on $0.2-\mu \mathrm{m}$ $47 \mathrm{~mm}$ polycarbonate filters (Poretics Corp, Livermore, CA, USA) and resuspended in respective sterile filtered water. Before adding the cells, we measured bacterial abundance in the microcosms and adjusted the added sample volume from the dispersal source to the original. Samples were taken 3 days after the respective dispersal events (Figure 2) (6 days after rewetting, on day 44, i.e., 'early rewetted sampling', and 2 weeks after rewetting, on day 55, i.e., 'late rewetted sampling', respectively) for the analysis of bacterial respiration, functional diversity, bacterial community composition, bacterial abundance, DOC and nutrient content, and conductivity measurements. The same analyses were also carried out for the dispersal source mix.

\section{Sample analysis}

Environmental parameters and microbial function. Conductivity, temperature, DOC and nutrient content (TP: total phosphorus; TN: total nitrogen) were determined as described before (Langenheder and Ragnarsson, 2007), while bacterial abundance (BA) was measured by flow cytometry according to Székely et al. (2013). Bacterial respiration rate (BR) was estimated as the rate of change of temperaturecompensated oxygen concentration measured for 3 days in airtight vessels using a needle-type oxygen microsensor (FTC) connected via an optical fibre to a Fibox 3 oxygen meter (PreSens, Regensburg, Germany). Cell-specific bacterial respiration (sBR) was calculated by normalizing BR to BA (sBR=BR/BA).

Heterotrophic functional diversity was estimated by measuring carbon substrate utilization profile using Biolog EcoPlatesTM (Biolog Inc., Hayward, CA, USA) and average colour development was calculated as described before (Berga et al., 2012, and references therein).

Active bacterial community composition. The composition of the active bacterioplankton was determined by 454-pyrosequencing according to the protocol described in Székely et al. (2013). Briefly, extracted 16S rRNA was reverse transcripted to 
exclude inactive bacteria with low ribosome content (Nikolausz et al., 2004). The amplicons were sequenced at the Norwegian Sequencing Centre (http://www.sequencing.uio.no) using GS-FLX Titanium chemistry (454 Life Sciences; Roche, Basel, Switzerland). A total of 413892 good quality sequences was obtained.

The sequences were processed using AmpliconNoise package and Perseus chimera removal program (Quince et al., 2011). The sequences were clustered into $3 \%$ dissimilarity operational taxonomic units (OTUs) using FCluster (http://fuzzy.cs. uni-magdeburg.de/fcluster/). OTUs were taxonomically identified with the naïve Bayesian classifier of Ribosomal Database Project (Wang et al., 2007) and only classifications above $80 \%$ bootstrap cutoff value were included in the analyses. Chloroplast OTUs and OTUs having only one sequence (i.e., singletons) were removed from the data set. Before statistical analyses, sequences were subsampled using the sequence number of the lowest sequence number sample. This way the samples were analysed based on the relative abundance of 964 OTUs with a minimum relative abundance of $0.365 \%$. All sequences used in this study have been deposited to the NCBI sequence read archive under accession number PRJEB17853.

\section{Data analysis}

Stress response groups and taxonomic analysis. OTUs were grouped based on their response towards the two main stress factors that comprise a dryingrewetting event (Figure 1): (1) the drying process, which challenges bacteria with the physical and chemical changes that arise due to the evaporation of water, and (2) the desiccated stage followed by rewetting, which OTUs can only cope with by forming desiccation withstanding dormant cells that successfully rescuccitate and recolonizing the water body after rewetting. To emphasize the difference in the response required to overcome the two different parts of the drying-rewetting stress, we defined the ability to withstand the drying process without change as resistance, and the capability to survive the transient phase of complete desiccation, and the ability to recover following rewetting, as tolerance (Brauner et al., 2016).

In the case of the drying stress, changes in relative abundance were interpreted as a measure of the stress response and used to categorize the OTUs. Namely, pairwise comparison ( $t$-test) of the relative abundance of each OTU in the control and the drying samples was performed, and (1) the OTUs that showed no significant difference in their relative abundance at the two sampling points were categorized as drying-resistant OTUs, whereas (2) those that had significantly lower abundance in the drying samples were grouped as drying-sensitive OTUs, and (3) those that had higher abundance at the drying sampling point were categorized as drying opportunists (Figure 1).

To better account for the stochastic nature of the processes involved in the coping with the desiccation-rewetting stress (i.e., formation of desiccation resistant dormant forms and successful awakening following rewetting), OTUs were categorized based on presence-absence at the different stages of the rewetting process (Figure 1). Namely, (1) rewettingtolerant OTUs were detected both before rewetting (drying and/or control) and in the early rewetted samples; (2) rewetting-sensitive OTUs that were active before the rewetting (either in the control or the drying treatment), but could not be detected in the early rewetted samples; and (3) rewettingopportunistic OTUs that could be detected only after rewetting (their relative abundance did not reach the detection limit of our study before the desiccation and rewetting, or were in those samples only inactive members of the local seed bank). Both drying and rewetting response groups were defined independently for each source pool.

The relative abundances of the most abundant phyla and classes $(\geqslant 1 \%$ mean relative abundance at any sampling point in the samples of at least one source pool) were also determined.

Assessing the predictability of the outcome of the dispersal treatments. The predicted outcome of the dispersal treatment in relationship to the relative abundance of the desiccation-rewetting response groups and specific respiration was calculated considering that all dispersed bacteria established with $100 \%$ efficacy in the recipient community. The predicted values of each variable $\left(V_{\mathrm{P}}\right)$ in the

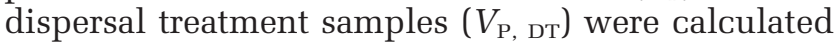
using the measured values of the respective variables $\left(V_{\mathrm{M}}\right)$ in the dispersal sources $\left(V_{\mathrm{M}, \mathrm{DS}}\right)$ and in the nondispersed samples $\left(V_{\mathrm{M}, \mathrm{ND}}\right)$, and the applied dispersal rates (dr) using the following formula: $V_{\mathrm{P}, \mathrm{DT}}=\mathrm{dr} \times$ $V_{\mathrm{M} \text {, DS }}+(1-\mathrm{dr}) \times V_{\mathrm{M}, \mathrm{ND}}$. The predicted values of the variables of the dispersal treatment $\left(V_{\mathrm{P}, \mathrm{DT}}\right)$ were then compared with the values measured in the samples that actually underwent the dispersal treatment $\left(V_{\mathrm{M}, \mathrm{DT}}\right)$ (see Statistical analysis). In case of no significant difference between predicted and observed values, the effect of dispersal was considered to be governed by neutral processes, whereas significant differences indicate that the establishment success of dispersed bacteria is influenced by species sorting processes.

Statistical analysis. To test the effects of the drying-rewetting process and the interaction of the effects with the source of the samples on the univariate variables two-way analyses of variance (ANOVA) with corresponding Tukey's post hoc tests were performed using sampling point (control, drying, early and late rewetted) and source pool (P, $\mathrm{M}, \mathrm{T}$ ) as the two grouping variables. The effect of the different dispersal rates on the univariate variables 
were analysed separately for the early and late dispersal treatments using two-way ANOVA in a similar way as for the drying-rewetting process but with dispersal rates (high, low or none) and source pool as grouping variables. The predictability of the establishment success of the different stress response groups and the respiration rates was tested for the variables of the low and high dispersal rate samples separately by comparing the predicted $\left(V_{\mathrm{P}, \mathrm{DT}}\right)$ and measured $\left(V_{\mathrm{M} \text {, пт }}\right)$ values and the interaction of their differences with the source of the samples using twoway ANOVA in a similar way as for the dryingrewetting process and the dispersal rates but with the generation of the value (predicted or measured) and source pool as grouping variables and dispersal rate as the blocking factor. Replicates (A, B and C) were used as randomized blocking factors in all linear models tested by ANOVA. To assure the fulfilment of the assumptions of ANOVA, normal distribution of the residuals of the linear models were tested using Shapiro-Wilk normality test, and the homogeneity of variance was checked using Levene's test and where needed, data were transformed.

Bacterial community composition and carbon substrate utilization were visualized using nonmetric multidimensional scaling based on BrayCurtis dissimilarities. Compositional and functional $\beta$-diversity at each sampling point was estimated based on multivariate dispersion ( $\beta$-dispersion) analysis (Anderson et al., 2006) using bacterial community composition or the substrate utilization profile of each replicate sample set (P, M and T). The effects of the drying-rewetting process and dispersal on the multivariate data were tested in a similar manner as for the univariate data but with two-way permutational multivariate analysis of variance (adonis function).

All statistical analyses and visualizations were performed using R. All multivariate data analyses were carried out with the vegan package (Oksanen et al., 2015), except for the Mantel tests that were conducted using the ecodist package (Goslee and Urban, 2007).

\section{Results}

Experimental conditions and bacterial abundance Conductivity, TN, TP, DOC and bacterial abundance changed significantly during the experiment $(P<0.001$; Supplementary Figure S1). At the beginning of the experiment, the three sources (P, M, T) showed relatively similar values for the measured environmental parameters with only some notable exceptions, such as slightly higher conductivity in the case of $\mathrm{P}$, or higher $\mathrm{TP}$ in the case of $\mathrm{T}$ (Supplementary Figures S1A and B). At the end of the drying phase (drying sampling) conductivity, $\mathrm{TN}, \mathrm{TP}$ and bacterial abundance increased significantly compared with the non-drying controls, which remained similar to the initial values
$(P<0.05$, exception: TP in the case of $\mathrm{P}$ samples; Supplementary Table S1 and Supplementary Figure S1). DOC also increased during the drying phase (Supplementary Figure S1D); however, an analogous increase was also detected in the control samples resulting in no significant difference between the drying and control samples $(P>0.1$; Supplementary Table S1D). After rewetting the environmental parameters in general returned to similar values than the ones measured in the non-drying control samples $(P>0.1$; Supplementary Table S1). Bacterial abundances followed similar trends with a rapid increase of abundance right after rewetting and a peak on day 41 (3 days after rewetting), irrespective of the source pool (Supplementary Figure S1E). At this time point, abundances were similar to those of the control samples at day 20 (control sampling) and was therefore chosen as the time point for the early dispersal treatment. At subsequent time points, abundances were almost always similar to the values of the non-drying control samples $(P>0.1$; Supplementary Table S1E).

\section{Effect of drying-rewetting process on bacterial communities}

Active bacterial community structure. Active bacterial community composition was significantly affected by the source pool of the samples, which alone explained $29-62 \%$ of variance of the samples (Figure 3a; permutational multivariate analysis of variance results: Supplementary Table S2). Similarly, the relative abundance of both the drying and the rewetting response groups were also significantly affected by the source pool (Figures 4 and 5). Regarding the drying-rewetting stress, community structure was clearly different before and after rewetting, whereas the community composition of the drying samples was only slightly different than the community composition of the controls, and the early and late stages after the rewetting showed also only minor differences (Figure 3a and Supplementary Table S2). Besides, compositional $\beta$-diversity did not change as a consequence of the drying stress (i.e., comparing control and drying samples) or from the early to the late rewetted sampling, but was significantly lower after rewetting (i.e., early and late together) than before the desiccation (i.e., control and drying samples) (Figure 3a, $P<0.05)$.

The changes in bacterial community composition through the drying-rewetting cycle were coupled to extensive turnover of OTUs as only OTUs corresponding to $11.5 \%$ relative abundance of the control communities were overall stress tolerant, meaning that they could be detected both at the end of the drying (drying sampling) and after the desiccationrewetting stress (early rewetted sampling). In addition, there were significant differences between the relative abundance of these overall tolerant OTUs 


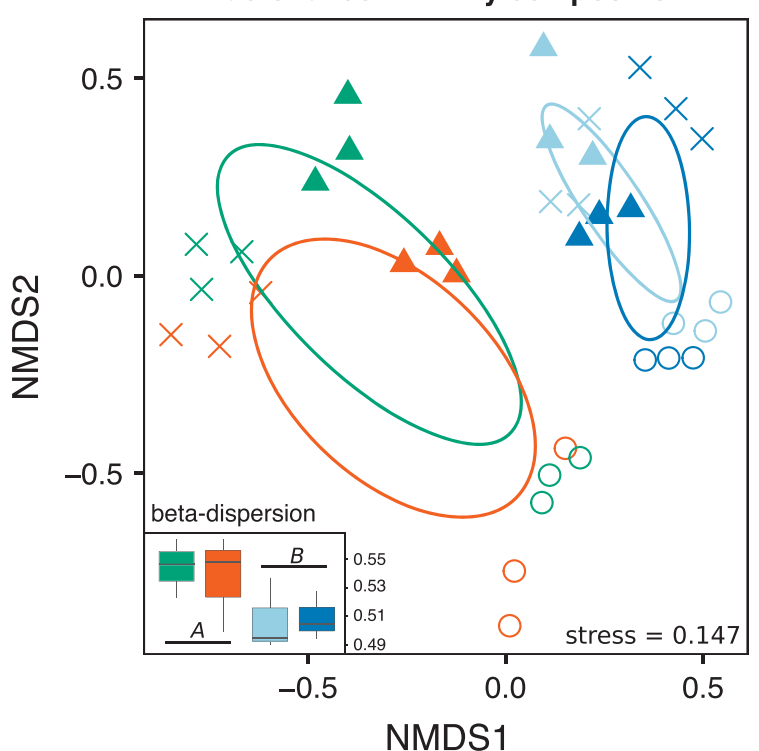

b

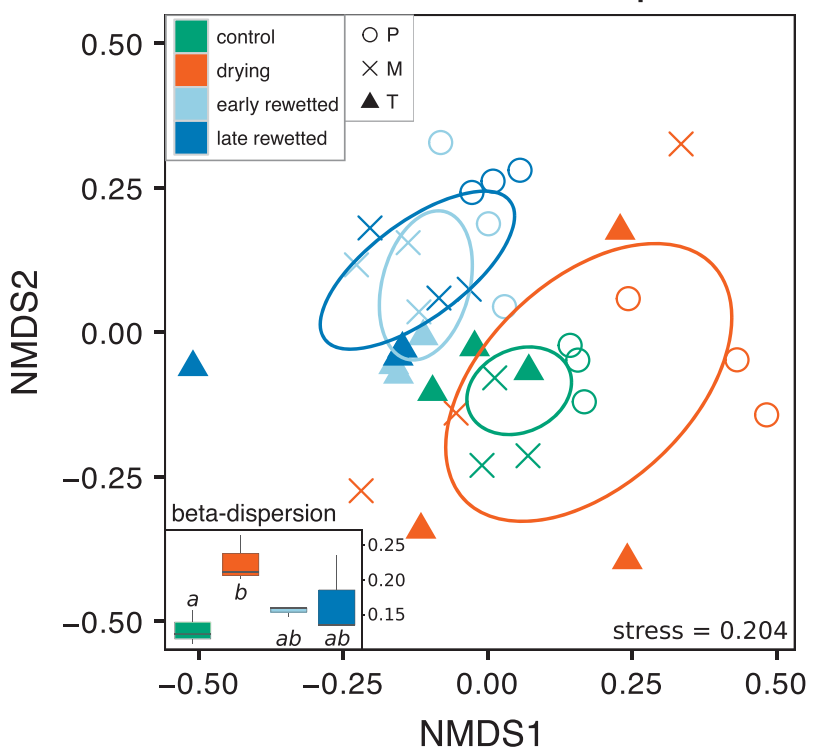

Figure 3 (a) Active bacterial community composition and (b) carbon substrate utilization profile visualized by non-metric multidimensional scaling (NMDS) and the corresponding $\beta$-dispersion values (small figures). On the NMDS plots, circles represent the permanent (P) pool samples, ' $\mathrm{x}$ ' the medium (M) and triangles the temporary $(\mathrm{T})$ pool samples; colour represents the different sampling points in both the small and big figures: green stands for control, red for drying, light blue for early rewetted and dark blue for late rewetted sampling. Ellipses represent the 95\% confidence ellipses of the samples of the corresponding sampling point. Significant differences $(P<0.05)$ in $\beta$-dispersal of the sampling points are represented in the small graphs by capital (before-after rewetting comparison) or lowercase letters (comparison of each sampling point).

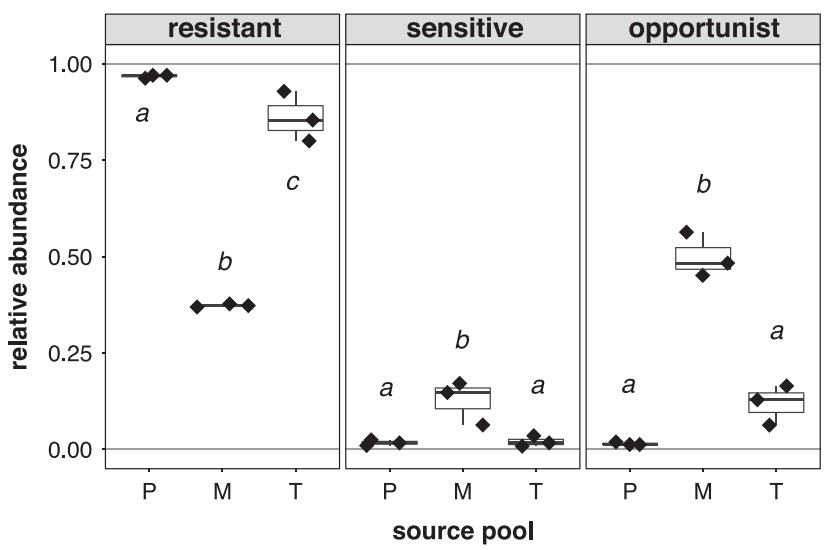

Figure 4 Differences in the relative abundances of the drying resistance groups in samples from different source pools at the drying sampling. Significant post hoc groups among the different pools are represented by lowercase letters.

between the control samples from the different sources (ANOVA: $F_{\text {source }}=8.33, P_{\text {source }}<0.05$ ), with the lowest relative abundances in $\mathrm{M}$ followed by $\mathrm{P}$ and then $\mathrm{T}(4.4 \pm 0.8 \%, 9.0 \pm 3.3 \%$ and $21.2 \pm 8.3 \%$, respectively).

The drying process did not have strong effects on bacterial community composition (Figure 3a) and most of the OTUs were drying resistant (Figure 4). However, the abundance of drying-resistant OTUs differed between pools, with $\mathrm{M}$ having significantly lower relative abundances of drying-resistant OTUs and higher abundances of drying-sensitive and -opportunistic OTUs than $\mathrm{P}$ and $\mathrm{T} \quad(P<0.001$, Figure 4). Interestingly, at the drying stage in the $\mathrm{M}$ samples, one single drying-opportunistic OTU (Zooglea sp.), which was completely absent from the control samples, became highly abundant and constituted half $(50 \pm 6 \%)$ of the community.

Before the desiccation and rewetting stages, rewetting-tolerant OTUs constituted only a minor part of the communities $(12.3 \pm 10.3 \%)$ and their relative abundance was not affected by the drying process (i.e., there was no significant difference in their relative abundance between the control and drying samples) $(P>0.1$, Figure 5). Early on after rewetting, the relative abundance of the rewettingtolerant OTUs increased significantly $(P<0.001)$, even though they remained only a minor part of the communities $(30.9 \pm 19.1 \%)$, whereas rewettingopportunistic OTUs (i.e., OTUs that were detected only after rewetting) became the most abundant members of the communities (Figure 5). Several differences could be observed between the samples of the different source pools with respect to the rewetting tolerance groups. Namely, before desiccation the $\mathrm{T}$ samples had significantly higher abundance of rewetting-tolerant OTUs than the samples of the other pools, while later after rewetting, M samples became completely dominated by rewetting-opportunistic OTUs, whereas $\mathrm{P}$ samples showed certain level of recovery to the conditions before the desiccation by the reappearance of rewetting-sensitive OTUs (Figure 5). 


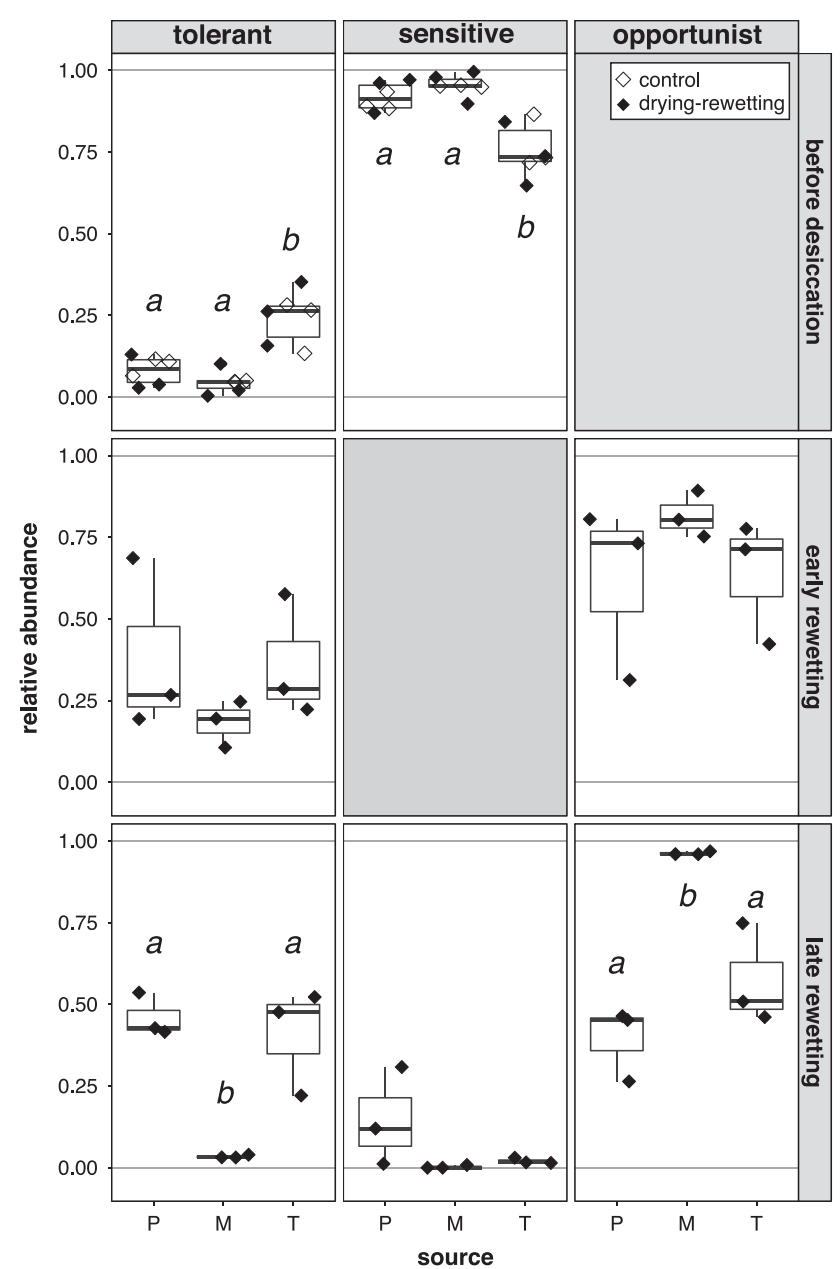

Figure 5 Differences in the relative abundances of the rewetting tolerance groups in samples from different source pools before desiccation (control and drying sampling), and early and late after rewetting. Empty rhomboids represent the samples of the control sampling, while the filled rhomboids represent the samples of the drying-rewetting sampling points (drying, early and late rewetted sampling). Significant post hoc groups $(P<0.05)$ among the different pools are represented by lowercase letters. Opportunist OTUs before desiccation and sensitive OTUs early after rewetting are not shown as those per se are absent from the given sampling points.

At the phylum and class level, characteristic trends were also apparent for all source pools (Supplementary Figure S2): after rewetting the average relative abundance of the Proteobacteria and Bacteroidetes together increased to $98 \pm 1.7 \%$ compared with the sampling points before rewetting $(69 \pm 21 \%$ ), whereas many previously common phyla and classes (e.g., Acidobacteria, Planctomycetes, Verrucomicrobia and Bacteroidia) decreased strongly in relative abundance. Characteristic differences in the relative abundances of different phylogenetic groups were also found for the different source pools in response to the drying and rewetting, respectively (Supplementary Figure S2).

Function. Specific bacterial respiration was significantly affected by the drying-rewetting stress (Supplementary Figure S1F). During the drying phase the difference between control and drying samples was only significant in the case of the $\mathrm{M}$ samples $(P<0.001$; Supplementary Table S1 and Supplementary Figure S1F). After rewetting respiration rates decreased in samples from all sources and were the lowest at the end of the experiment (Supplementary Table S1F and Supplementary Figure S1F).

The carbon substrate utilization profile also showed a succession through the drying-rewetting process with clear separation between the samples from before (i.e., control and drying) and after rewetting (early and late rewetted) (Figure $3 \mathrm{~b}$ and Supplementary Table S3A). In addition, differences between the source pools were less pronounced than for the community composition (Figure $3 \mathrm{~b}$ and Supplementary Tables S2 and S3). However, there were more pronounced differences in carbon substrate utilization between the drying and control treatment than in case of community composition and the substrate utilization-derived functional $\beta$ diversity was significantly higher in the drying samples compared with the control treatment.

Effects of dispersal rates and timing during the rewetted phase

Effect of dispersal on bacterial communities. Dispersal treatment did not influence bacterial community composition when it occurred later after rewetting but caused marginally significant changes in community composition when it occurred early after rewetting (variance explained by dispersal: early rewetting= $6.7 \%, P<0.1$; late rewetting variance explained $=3.7 \%$; not significant) (Supplementary Table S4). However, both the early and late dispersal treatments significantly increased the relative abundance of rewettingsensitive OTUs and decreased the relative abundance of rewetting-opportunistic OTUs, which enhanced conversion towards the conditions before desiccation (Figures $6 \mathrm{~b}$ and $\mathrm{c}$ and Supplementary Table S5). In addition, dispersal also had an effect on phyla composition as it reintroduced phyla such as Planctomycetes or Verrucomicrobia that were eliminated after desiccation and rewetting (Supplementary Figure S2). The effect of dispersal on carbon substrate utilization was significant in the case of both the early and late treatment, but it was more pronounced in the case of the early dispersal treatment (variance explained by dispersal: early rewetting $=13.5 \%, P<0.01$; late rewetting $=8.4 \%, P<0.05$ ) (Supplementary Table S4). Respiration rates significantly increased towards predesiccation values as a consequence of the dispersal treatment in the early but not in the late treatment (Figure 6d and Supplementary Table S5).

Effect of dispersal timing on bacterial communities. The comparison of the predicted and measured relative abundances of the rewetting tolerance groups that were significantly modified by the dispersal treatments showed that more rewettingsensitive and less rewetting-opportunistic bacteria 


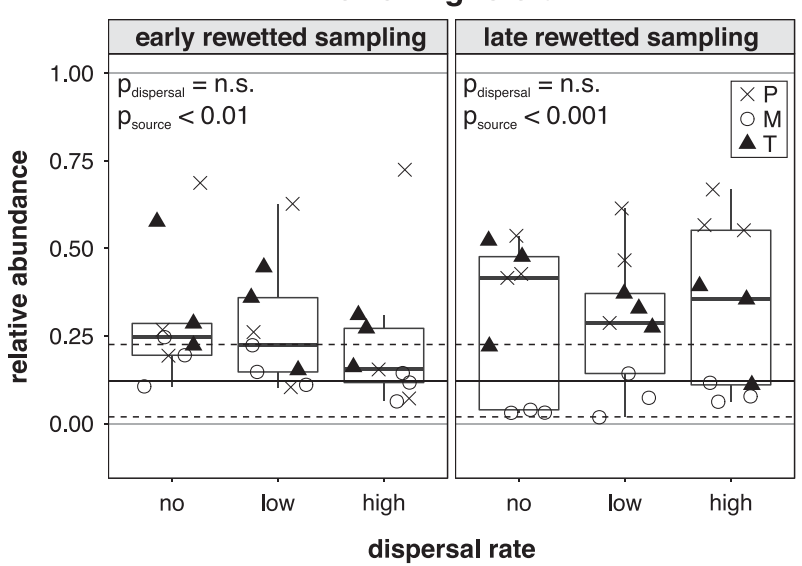

C

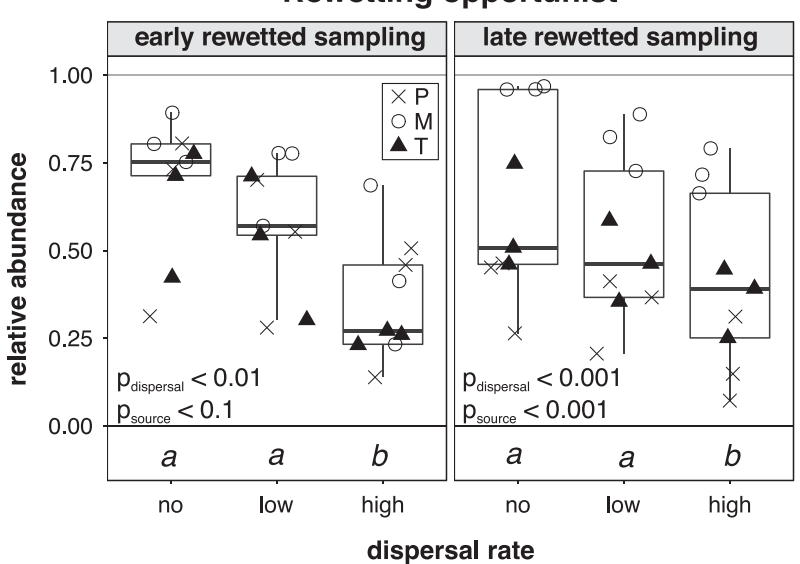

b

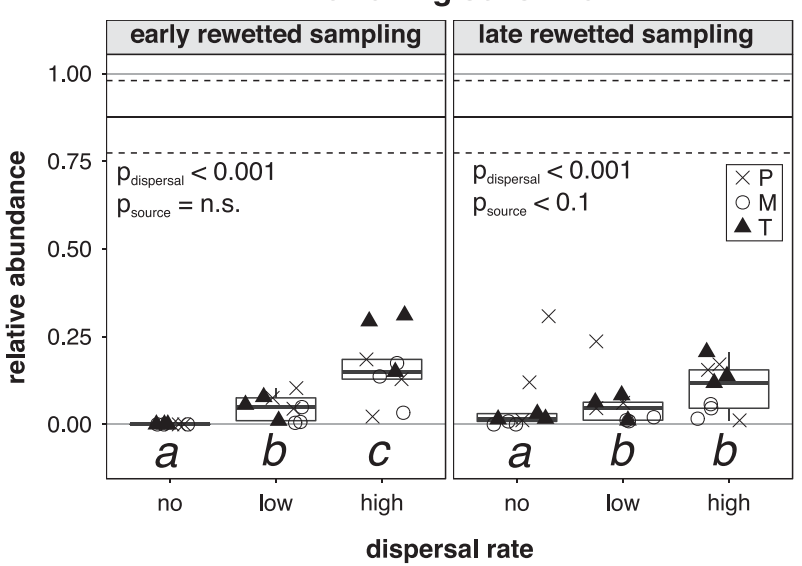

d

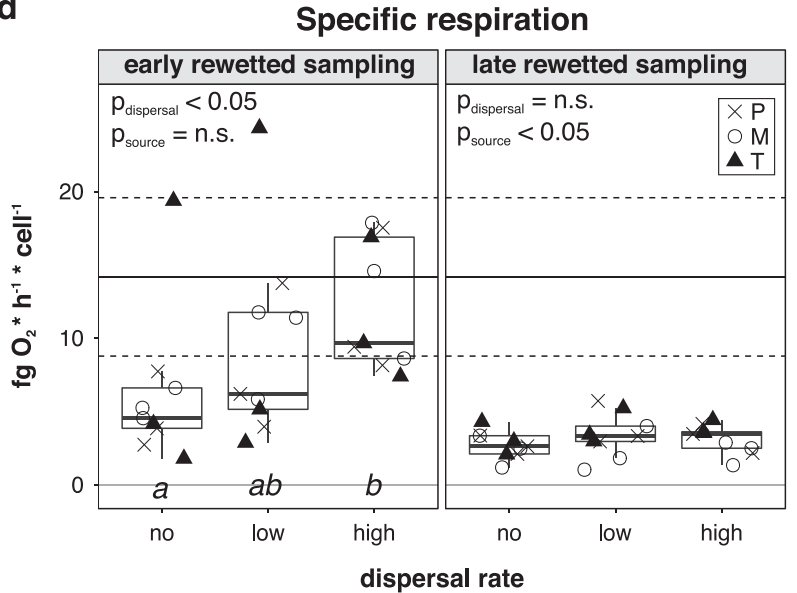

Figure 6 The effect of early and late dispersal treatments on the relative abundances of rewetting tolerance groups (a-c) and respiration rates (d). (a) Rewetting tolerant; (b) rewetting sensitive; (c) rewetting opportunistic; (d) specific respiration. The different symbols represent the different source pools (empty circles indicate the permanent $(\mathrm{P})$, ' $\mathrm{x}$ ' indicate the medium $(\mathrm{M})$ and triangles the temporary ( $\mathrm{T}$ ) pool). Solid horizontal lines indicate the mean values of the given parameter in the samples before desiccation (control and drying sampling), whereas dashed lines indicate the standard deviation from the mean values. Significant post hoc groups $(P<0.05)$ among the different dispersal treatment levels (no, low, high rate) are represented by lowercase letters. The $P$-values of corresponding two-way ANOVAs are shown on the figures (for more see Supplementary Table S5).

established in the dispersed communities in the early dispersal treatment than predicted, indicating that the outcome of the dispersal treatment was not predictable based on the dispersal rate (Figures $7 \mathrm{a}$ and b and Supplementary Table S6). Meanwhile, in the late dispersal treatment samples, only the measured abundance of the opportunistic OTUs deviated from the predicted values. The comparison of measured and predicted respiration rates showed similar trends than the rewetting tolerance groups, with the early dispersal treatment resulting in higher respirations rates than predicted, while the respiration of late treatment samples was as predicted, indicating a more dispersal rate-dependent dispersal treatment outcome (Figure 7c).

\section{Discussion}

In this study, we were able to simulate the physical and chemical changes that have been demonstrated for drying and rewetting events in natural water bodies (Boven et al., 2008; Vazquez et al., 2010; Ylla et al., 2010; Zoppini et al., 2010) under controlled laboratory circumstances. First, we show that the extent of the bacterial community changes provoked by the stress event, as well as the subsequent recovery, was influenced by the initial conditions and the history of the communities, that is, the frequency of previous drying-rewetting cycles. Second, we demonstrate that dispersal promoted recovery to the state before the stress episode by reintroducing sensitive taxa, and promoting functional recovery. Finally, we show that the timing of the dispersal event modified the establishment success of dispersed bacteria, resulting in varying predictability of the outcome of dispersal, and consequently the degree by which dispersal facilitated recovery.

As expected, the drying process led to elevated levels of salinity (conductivity) and nutrient concentration in all incubations (Supplementary 


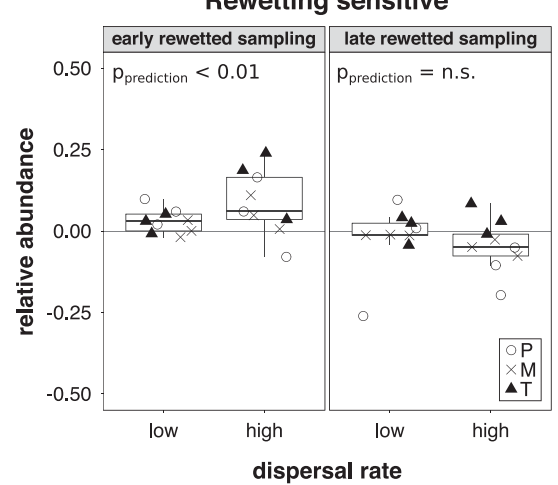

b

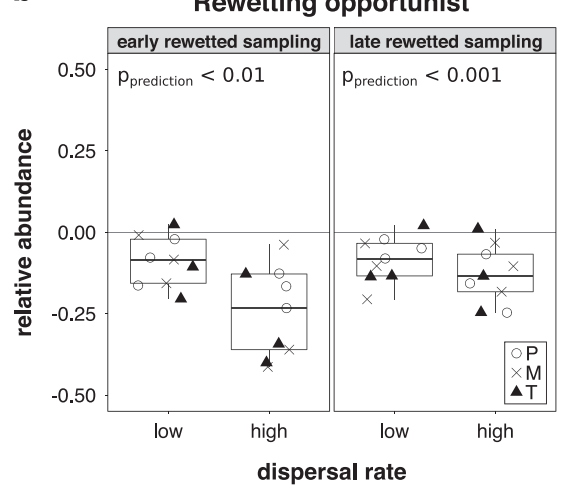

C

Figure 7 Differences between predicted and measured values of the rewetting tolerance groups (a and $\mathbf{b}$ ) and specific respiration rates (c). Only tolerance groups that were significantly affected by the dispersal treatments are shown. Positive values indicate that the measured values were higher than the predicted values $\left(V_{\mathrm{M}}>V_{\mathrm{P}}\right)$, whereas negative values indicate the opposite $\left(V_{\mathrm{M}}<V_{\mathrm{P}}\right)$. (a) Rewetting sensitive; (b) rewetting opportunistic; (c) specific respiration). Empty circles: permanent pool (P); ' $\mathrm{x}$ ': medium (M) pool; triangles: temporary (T) pool. The $P$-values of corresponding ANOVAs are shown on the figures (for more see Supplementary Table S6).

Figure S1), which, however, did not result in strong changes in bacterial community composition, indicating compositional resistance during the drying process (Figure 3a). This was surprising since other studies have shown that minor changes in conductivity and nutrient concentration modify bacterial community structure (Lozupone and Knight, 2007; Berga et al., 2012; Székely and Langenheder, 2014; Zeglin, 2015). It is possible that this is the result of long-term adaptation to environmental variations, including changes in salinity and nutrient concentration that are characteristic for the studied rock pools (Jocqué et al., 2010; Langenheder et al., 2012). Compared with composition, carbon substrate utilization profiles changed more clearly during the drying phase and also induced an increase in functional $\beta$-diversity (Figure 3b). Functional divergence as a response to environmental change without corresponding changes in composition indicates functional plasticity, and implies high phenotypic plasticity of the given bacteria (Beier et al., 2015). On the other hand, specific respiration only increased as a consequence of the drying process in the case of the $\mathrm{M}$ samples, which also changed substantially in composition (i.e., had the least abundance of dryingresistant OTUs) (Figure 4), indicating that changes in this functional parameter were coupled with compositional changes.

The effect of complete desiccation and rewetting on bacterial community composition differed clearly from the effect of the drying process. Although after rewetting most of the chemical parameters resembled the conditions of the initial samples and the controls, there were clear differences in bacterial community composition (Figure 3a) and communities were dominated by rewetting-opportunistic OTUs (Figure 5). Similar dynamics have been recently demonstrated when comparing the communities of dry and rewetted soils: $69-74 \%$ of the taxa in the rewetted samples were not detected in the dry soils (Aanderud et al., 2015). The authors argued that these newly emerged taxa were either previously dormant and were resuscitated by moisture or had previously low abundance and proliferated in response to the environmental cue. In our case, where an aquatic community re-emerged after desiccation and rewetting, it seems likely that when the vast majority of the previous communities was filtered out, niches opened for previously rare, opportunistic OTUs from seed banks to proliferate (Shade et al., 2014). In addition, $\beta$-diversity decreased as a result of the desiccation-rewetting process (Figure 3a), which is in congruence with results from macroinvertebrate communities in ponds that experienced drought (Chase, 2007). This indicates that despite the stochasticity of the entry and rescussitation from dormancy (Beaumont et al., 2009; Buerger et al., 2012) and the divergence of communities anticipated following an inactive period (Lee et al., 2017), desiccation and rewetting stress homogenizes communities as expected for high-intensity disturbances (Jiang and Patel, 2008). Moreover, the desiccation and rewetting also led to the disappearance of several phyla, which implies that the traits necessary for desiccation and rewetting resistance (e.g., formation of desiccation resistant dormant cells) are conserved at broad taxonomic levels (Evans and Wallenstein, 2014; Martiny et al., 2015; Amend et al., 2016) (Supplementary Figure S2). Compositional resilience (i.e., recovery of composition similar to that of the communities preceeding the desiccation-rewetting stress) was not observed (Figure 3a), not even after prolonged incubation (late rewetted samples), further reinforcing that communities can not readily recover from the harshness of the drought event. Interestingly, before desiccation, the drying process did not prime the rewetting-tolerant or -sensitive OTUs to 'prepare' for the desiccation phase as there was no difference in their abundance between the control and drying samples (Figure 5).

Rewetting also led to clear changes in functional parameters compared with samples taken before the desiccation and rewetting event and there was no 
functional recovery (Figure 3b and Supplementary Figure S1F). The lack of recovery was surprising because microbial communities are often considered to be functionally redundant (Allison and Martiny, 2008; Nielsen et al., 2011) so that the functional performance of the rewetted communities would resemble that of the control communities despite compositional differences. Moreover, we found that even respiration, that is, a general singular functional parameter, that is carried out by many species, and therefore regarded as robust to disturbances and environmental changes (Gamfeldt et al., 2007; Peter et al., 2011), was affected by the desiccationrewetting event. This emphasizes the harshness of this disturbance and points out the importance of qualitative assessments of stressors when predicting their disrupting effect on ecosystems.

Although similar trends in the response to the drying-rewetting stress were found for all source pools, there were also substantial differences, which potentially reflected differences in the drought history of the pools. Based on the hydrological history of the pools, we expected that communities in the permanent pool (P) should be the most sensitive, followed by the intermediate pool (M), whereas the temporary pool (T) was expected to be the most resistant. Instead, the intermediate pool (M) was the least resistant, which was reflected by the lowest abundance of drying-resistant as well as overall (i.e, both drying and rewetting) tolerant OTUs in the drying and control samples, respectively, and by the lowest level of recovery following rewetting (Figures 4 and 5). Meanwhile, $\mathrm{P}$ showed similar resistance to the stress event than $\mathrm{T}$, with slightly higher abundances of drying-resistant OTUs and recovery of rewetting-sensitive OTUs, and lower initial rewetting-tolerant and overall tolerant OTU abundance (Figures 4 and 5). We can only speculate about the possible reasons for this unexpected result, but they may include (a) the lack of knowledge of the history of other environmental stress events (e.g., salinity changes due to sea spray input), (b) differences in the original environmental parameters and consequently the community composition of the pools. Namely, P-presumably due to sea spray input-had slightly higher initial conductivity than $\mathrm{M}$ and $\mathrm{T}$, and was dominated by AlphaProteobacteria, a class known for being abundant in environments with elevated conductivity (Newton et al., 2011). Hence, this might have increased the resistance and recovery capacities of this community towards stress events encompassing conductivity changes. Such difference in the stress response depending on the disturbance history or previous conditions of the given microbial community (i.e., stress priming) is common in microbial communities and particularly strong for stressors that involve osmotic changes (Andrade-Linares et al., 2016). Hence, our results show that the extent of the effect of both parts of the stress episode is influenced by the initial conditions and stress history of the communities, with some indications that the more 'battle-hardened' communities are the most resistant.

Dispersal did not substantially change the composition of bacterial communities (Supplementary Table S4), but it successfully reintroduced rewetting-sensitive OTUs and phyla that were filtered out by the rewetting process (Figures 6a-c and Supplementary Figure S2). Dispersal also had pronounced effects on the functional potential of the communities (Figure 6d and Supplementary Table S4). This deviating response between function and overall community composition suggests that dispersal had a 'rescue effect' and introduced key community members that were substantial with regard to the assessed functions (Nielsen et al., 2011), and emphasizes the importance of dispersal in the recovery from harsh stress events. However, the effect of the dispersal events depended on their timing, that is, whether they occurred early or later after rewetting. Generally, the effects were much stronger when dispersal occurred during the early phase of the community reassembly process (Figure 6 and Supplementary Table S4). At this stage, the establishment success of rewettingsensitive bacteria was higher than predicted based on the dispersal rates (Figure 7), which supports evidence for strong deterministic effects of dispersal at early stages of community development (Vellend et al., 2014). This suggests that species sorting processes related to the environmental conditions and interactions within the recipient community had strong effects when dispersal occurred at an early stage after the rewetting, whereas effects of dispersal were neutral, that is, predictable based on dispersal rates, at the later stage. The importance of colonization timing in dispersal processes has been shown before in single-species systems (e.g., Fukami et al., 2007) and here we provide now clear evidence that it may be important in the establishment of complex dispersed communities and their role in the recovery of the recipient communities following disturbances.

In summary, our study shows that information about the stress history and dispersal and its timing in relation to the successional state of a local community are essential to understand and predict the response of bacterial communities to environmental stress.

\section{Conflict of Interest}

The authors declare no conflict of interest.

\section{Acknowledgements}

We thank Mercè Berga for her help during field sampling, Daniel Lundin for assistance with sequence data processing and Tamás Felföldi for providing the expanded-clay biofilm carriers. This research was supported by the Carl Tryggers foundation, and the Marie Curie International Outgoing Fellowship within the Seventh European Community Framework Programme. 


\section{References}

Aanderud ZT, Jones SE, Fierer N, Lennon JT. (2015). Resuscitation of the rare biosphere contributes to pulses of ecosystem activity. Front Microbiol 6: 24.

Allison SD, Martiny JBH. (2008). Resistance, resilience, and redundancy in microbial communities. Proc Natl Acad Sci USA 105: 11512-11519.

Amalfitano S, Fazi S, Zoppini A, Barra Caracciolo A, Grenni P, Puddu A. (2008). Responses of benthic bacteria to experimental drying in sediments from Mediterranean temporary rivers. Microb Ecol 55: 270-279.

Amend AS, Martiny AC, Allison SD, Berlemont R, Goulden ML, Lu Y et al. (2016). Microbial response to simulated global change is phylogenetically conserved and linked with functional potential. ISME J10: 109-118.

Anderson MJ, Ellingsen KE, McArdle BH. (2006). Multivariate dispersion as a measure of beta diversity. Ecol Lett 9: 683-693.

Andrade-Linares DR, Lehmann A, Rillig MC. (2016). Microbial stress priming: a meta-analysis. Environ Microbiol 18: 1277-1288.

Barthès A, Ten-Hage L, Lamy A, Rols J-L, Leflaive J. (2015). Resilience of aggregated microbial communities subjected to drought-small-scale studies. Microb Ecol 70: 9-20.

Beaumont HJE, Gallie J, Kost C, Ferguson GC, Rainey PB. (2009). Experimental evolution of bet hedging. Nature 462: 90-93.

Beier S, Rivers AR, Moran MA, Obernosterer I. (2015). Phenotypic plasticity in heterotrophic marine microbial communities in continuous cultures. ISME $J$ 9: 1141-1151.

Berga M, Székely AJ, Langenheder S. (2012). Effects of disturbance intensity and frequency on bacterial community composition and function. PLoS One 7: e36959.

Bogan MT, Lytle DA. (2011). Severe drought drives novel community trajectories in desert stream pools. Freshw Biol 56: 2070-2081.

Boven L, Stoks R, Brendonck L, Biology E, Natural H, Museum H. (2008). Seasonal dynamics in water quality and vegetation cover in temporary pools with variable hydroperiods in Kiskunság (Hungary). Wetlands 28: 401-410.

Brauner A, Fridman O, Gefen O, Balaban NQ. (2016). Antibiotic treatment. Nat Publ Gr 14: 320-330.

Bressan M, Mougel C, Dequiedt S, Maron P-A, Lemanceau P, Ranjard L. (2008). Response of soil bacterial community structure to successive perturbations of different types and intensities. Environ Microbiol 10: 2184-2187.

Brock MA, Nielsen DL, Shiel RJ, Green JD, Langley JD. (2003). Drought and aquatic community resilience: the role of eggs and seeds in sediments of temporary wetlands. Freshw Biol 48: 1207-1218.

Buerger S, Spoering A, Gavrish E, Leslin C, Ling L, Epstein SS. (2012). Microbial scout hypothesis, stochastic exit from dormancy, and the nature of slow growers. Appl Environ Microbiol 78: 3221-3228.

Chase JM. (2007). Drought mediates the importance of stochastic community assembly. Proc Natl Acad Sci USA 104: 17430-17434.

Comte J, del Giorgio PA. (2011). Composition influences the pathway but not the outcome of the metabolic response of bacterioplankton to resource shifts. PLoS ONE 6: e25266.

Evans TG, Hofmann GE. (2012). Defining the limits of physiological plasticity: how gene expression can assess and predict the consequences of ocean change. Philos Trans $R$ Soc Ser B 367: 1733-1745.

Evans SE, Wallenstein MD. (2014). Climate change alters ecological strategies of soil bacteria. Ecol Lett 17: 155-164.

Fazi S, Amalfitano S, Piccini C, Zoppini A, Puddu A, Pernthaler J. (2008). Colonization of overlaying water by bacteria from dry river sediments. Environ Microbiol 10: 2760-2772.

Fazi S, Vázquez E, Casamayor EO, Amalfitano S, Butturini A. (2013). Stream hydrological fragmentation drives bacterioplankton community composition. PLoS One 8: e64109.

Fierer N, Nemergut D, Knight R, Craine JM. (2010). Changes through time: Integrating microorganisms into the study of succession. Res Microbiol 161: 635-642.

Fukami T. (2015). Historical contingency in community assembly: integrating niches, species pools, and priority effects. Annu Rev Ecol Evol Syst 46: 1-23.

Fukami T, Beaumont HJE, Zhang X-X, Rainey PB. (2007). Immigration history controls diversification in experimental adaptive radiation. Nature 446: 436-439.

Gamfeldt L, Hillebrand H, Jonsson PR. (2007). Multiple functions increase the importance of biodiversity for overall ecosystem functioning. Ecology 88: 1622-1633.

Goslee SC, Urban DL. (2007). The ecodist package for dissimilarity-based analysis of ecological data. J Stat Softw 22: 1-19.

Griffiths BS, Philippot L. (2013). Insights into the resistance and resilience of the soil microbial community. FEMS Microbiol Rev 37: 112-129.

Hanson CA, Fuhrman JA, Horner-Devine MC, Martiny JBH. (2012). Beyond biogeographic patterns: processes shaping the microbial landscape. Nat Rev Microbiol 10: $497-506$.

Hawkes CV, Keitt TH. (2015). Resilience vs. historical contingency in microbial responses to environmental change. Ecol Lett 18: 612-625.

Hubbell SP. (2001). The Unified Neutral Theory of Biodiversity and Biogeography. Princeton University Press: Princeton, NJ, USA.

Humphries P, Baldwin DS. (2003). Drought and aquatic ecosystems: an introduction. Freshw Biol 48: 11411146.

Jiang L, Patel SN. (2008). Community assembly in the presence of disturbance: a microcosm experiment. Ecology 89: 1931-1940.

Jiménez Cisneros BE, Oki T, Arnell NW, Benito G, Cogley JG, Döll P et al. (2014). Freshwater resources. In: Field CB, Barros VR, Dokken DJ, Mach KJ, Mastrandrea MD, Bilir TE et al. (eds). Climate Change 2014: Impacts, Adaptation, and Vulnerability. Part A: Global and Sectoral Aspects. Contribution of Working Group II to the Fifth Assessment Report of the Intergovernmental Panel of Climate Change. Cambridge University Press: Cambridge, UK and New York, NY, USA, pp 229-269.

Jocqué M, Vanschoenwinkel B, Brendonck L. (2010). Freshwater rock pools: a review of habitat characteristics, faunal diversity and conservation value. Freshw Biol 55: 1587-1602. 
Lake PS. (2003). Ecological effects of perturbation by drought in flowing waters. Freshw Biol 48: 11611172 .

Langenheder S, Berga M, Östman Ö, Székely AJ. (2012). Temporal variation of $\beta$-diversity and assembly mechanisms in a bacterial metacommunity. ISME J 6: 1107-1114.

Langenheder S, Ragnarsson H. (2007). The role of environmental and spatial factors for the composition of aquatic bacterial communities. Ecology 88: 2154-2161.

Lee S, Sorensen JW, Grady KL, Tobin TC, Shade A. (2017). Divergent extremes but convergent recovery of bacterial and archaeal soil communities to an ongoing subterranean coal mine fire. ISME J. 1-13.

Lennon JT, Jones SE. (2011). Microbial seed banks: the ecological and evolutionary implications of dormancy. Nat Rev Microbiol 9: 119-130.

Lozupone CA, Knight R. (2007). Global patterns in bacterial diversity. Proc Natl Acad Sci USA 104: 11436-11440.

Mallon CA, Van Elsas JD, Salles JF. (2015). Microbial invasions: the process, patterns, and mechanisms. Trends Microbiol 23: 719-729.

Martiny JBH, Jones SE, Lennon JT, Martiny AC. (2015). Microbiomes in light of traits: a phylogenetic perspective. Science 350: aac9323.

Marxsen J, Zoppini A, Wilczek S. (2010). Microbial communities in streambed sediments recovering from desiccation. FEMS Microbiol Ecol 71: 374-386.

Newton RJ, Jones SE, Eiler A, McMahon KD, Bertilsson S. (2011). A guide to the natural history of freshwater lake bacteria. Microbiol Mol Biol Rev 75: 14-49.

Nielsen UN, Ayres E, Wall DH, Bardgett RD. (2011). Soil biodiversity and carbon cycling: a review and synthesis of studies examining diversity-function relationships. Eur J Soil Sci 62: 105-116.

Nikolausz M, Márialigeti K, Kovács G. (2004). Comparison of RNA- and DNA-based species diversity investigations in rhizoplane bacteriology with respect to chloroplast sequence exclusion. J Microbiol Methods 56: 365-373.

Oksanen AJ, Blanchet FG, Kindt R, Legendre P, Minchin PR, Hara RBO et al. (2015). Community Ecology Package: Vegan. Version 2.3-0. Available from: http://r-forge.rproject.org/projects/vegan/.

Östman Ö, Drakare S, Kritzberg ES, Langenheder S, Logue JB, Lindström ES. (2010). Regional invariance among microbial communities. Ecol Lett 13: 118-127.

Peter H, Ylla I, Gudasz C, Romaní AM, Sabater S, Tranvik LJ. (2011). Multifunctionality and diversity in bacterial biofilms. PLoS One 6: e23225.

Pohlon E, Ochoa Fandino A, Marxsen J. (2013). Bacterial community composition and extracellular enzyme activity in temperate streambed sediment during drying and rewetting. PLoS One 8: e83365.

Proia L, Vilches C, Boninneau C, Kantiani L, Farré M, Romaní AM et al. (2013). Drought episode modulates the response of river biofilms to triclosan. Aquat Toxicol 127: 36-45.
Quince C, Lanzen A, Davenport RJ, Turnbaugh PJ. (2011). Removing noise from pyrosequenced amplicons. BMC Bioinform 12: 38.

Schimel J, Balser TC, Wallenstein M. (2007). Microbial stress-response physiology and its implications. Ecology 88: 1386-1394.

Shade A, Jones SE, Caporaso JG, Handelsman J, Knight R, Fierer $\mathrm{N}$ et al. (2014). Conditionally rare taxa disproportionately contribute to temporal changes in microbial diversity. MBio 5: e01371-14.

Shade A, Peter H, Allison SD, Baho DL, Berga M, Bürgmann $\mathrm{H}$ et al. (2012). Fundamentals of microbial community resistance and resilience. Front Microbiol 3: 417.

Sloan WT, Lunn M, Woodcock S, Head IM, Nee S, Curtis TP. (2006). Quantifying the roles of immigration and chance in shaping prokaryote community structure. Environ Microbiol 8: 732-740.

Székely AJ, Berga M, Langenheder S. (2013). Mechanisms determining the fate of dispersed bacterial communities in new environments. ISME J 7: 61-71.

Székely AJ, Langenheder S. (2014). The importance of species sorting differs between habitat generalists and specialists in bacterial communities. FEMS Microbiol Ecol 87: 102-112.

Timoner X, Acuña V, Frampton L, Pollard P, Sabater S, Bunn SE. (2014). Biofilm functional responses to the rehydration of a dry intermittent stream. Hydrobiologia 727: 185-195.

Timoner X, Acuña V, Von Schiller D, Sabater S. (2012). Functional responses of stream biofilms to flow cessation, desiccation and rewetting. Freshw Biol 57: 1565-1578.

Vazquez E, Amalfitano S, Fazi S, Butturini A. (2010). Dissolved organic matter composition in a fragmented Mediterranean fluvial system under severe drought conditions. Biogeochemistry 102: 59-72.

Vellend M, Srivastava DS, Anderson KM, Brown CD, Jankowski JE, Kleynhans EJ et al. (2014). Assessing the relative importance of neutral stochasticity in ecological communities. Oikos 123: 1420-1430.

Wang Q, Garrity GM, Tiedje JM, Cole JR. (2007). Naive Bayesian classifier for rapid assignment of rRNA sequences into the new bacterial taxonomy. Appl Environ Microbiol 73: 5261-5267.

Ylla I, Sanpera-Calbet I, Vázquez E, Romaní AM, Muñoz I, Butturini A et al. (2010). Organic matter availability during pre- and post-drought periods in a Mediterranean stream. Hydrobiologia 657: 217-232.

Zeglin LH. (2015). Stream microbial diversity in response to environmental changes: review and synthesis of existing research. Front Microbiol 6: 454.

Zoppini A, Amalfitano S, Fazi S, Puddu A. (2010). Dynamics of a benthic microbial community in a riverine environment subject to hydrological fluctuations (Mulargia River, Italy). Hydrobiologia 657: 37-51.

Supplementary Information accompanies this paper on The ISME Journal website (http://www.nature.com/ismej) 